\title{
Foundation XML for Flash
}

Sas Jacobs

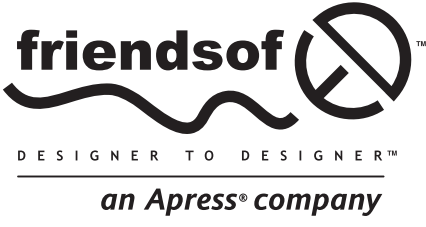




\title{
Foundation XML for Flash
}

\author{
Copyright (C) 2006 by Sas Jacobs
}

All rights reserved. No part of this work may be reproduced or transmitted in any form or by any means, electronic or mechanical, including photocopying, recording, or by any information storage or retrieval system, without the prior written permission of the copyright owner and the publisher.

ISBN (pbk): 1-59059-543-2

Printed and bound in the United States of America 987654321

Distributed to the book trade worldwide by Springer-Verlag New York, Inc., 233 Spring Street, 6th Floor, New York, NY 10013.

Phone 1-800-SPRINGER, fax 201-348-4505, e-mail orders-ny@springer-sbm.com, or visit www.springeronline.com.

For information on translations, please contact Apress directly at 2560 Ninth Street, Suite 219, Berkeley, CA 94710.

Phone 510-549-5930, fax 510-549-5939, e-mail info@apress.com, or visit www.apress.com.

The information in this book is distributed on an "as is" basis, without warranty. Although every precaution has been taken in the preparation of this work, neither the author(s) nor Apress shall have any liability to any person or entity with respect to any loss or damage caused or alleged to be caused directly or indirectly by the information contained in this work.

The source code for this book is freely available to readers at ww. friendsofed.com in the Source Code section.

\section{Credis}

Lead Editor Assistant Production Director

Chris Mills Kari Brooks-Copony

Technical Reviewer

Production Editor

Kevin Ruse

Kelly Winquist

Editorial Board

Steve Anglin, Dan Appleman Ewan Buckingham, Gary Cornell

Tony Davis, Jason Gilmore

Jonathan Hassell, Chris Mills

Dominic Shakeshaft, Jim Sumser

Associate Publisher

Grace Wong

Project Manager

Pat Christenson

Copy Edit Manager

Nicole LeClerc

Copy Editor

Compositor

Katy Freer

Proofreader

Lori Bring

Indexer

Broccoli Information Management

Artist

Katy Freer

\section{Cover Designers}

Corné van Dooren, Kurt Krames

\section{Manufacturing Director}

Tom Debolski 
For my parents, David and Sherry-Anne, and my sister, Lucy. Thanks for all your support. I feel lucky to have been born into such a terrific family. 


\section{CONTENTS AT A GLANCE}

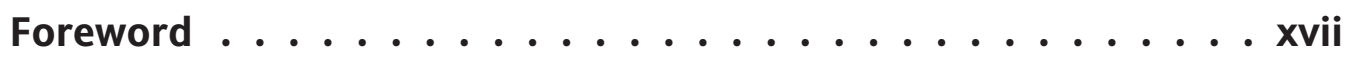

About the Author ................ . . . . . . .

About the Technical Reviewer ............. . xx

About the Cover Image $\ldots \ldots \ldots$............ xxi

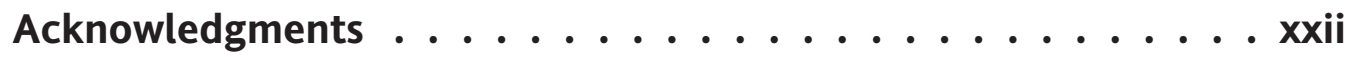

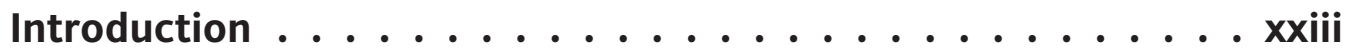

Chapter 1: Flash and XML ...............

Chapter 2: Introduction to XML ............. 19

Chapter 3: XML Documents . . . . . . . . . . . 57

Chapter 4: Using the XML Class . . . . . . . . . . . . . . 125

Chapter 5: Working with XML in Word $2003 \ldots . . . . . .217$

Chapter 6: Working with XML in Excel 2003 . . . . . . . . 257

Chapter 7: Working with XML in Access 2003 . . . . . . . . . 291

Chapter 8: Using the Data Components with XML . . . . . . 325

Chapter 9: Consuming Web Services with Flash . . . . . . . . 367

Chapter 10: Using the XMLSocket Class . . . . . . . . 411

Chapter 11: Which XML Option Is Best for Me? . . . . . . . . 427

Appendix: Useful Online Resources . . . . . . . . . . . . . 439

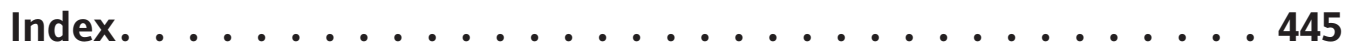




\section{CONTENTS}

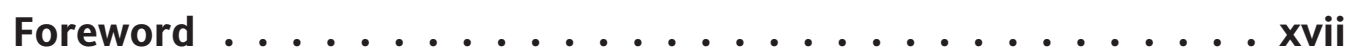

About the Author ................ xix

About the Technical Reviewer ............. . xx

About the Cover Image $\ldots \ldots \ldots$ xxi

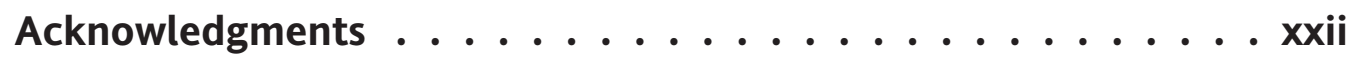

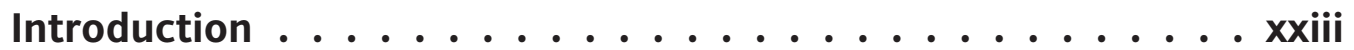

Chapter 1: Flash and XML ............... 1

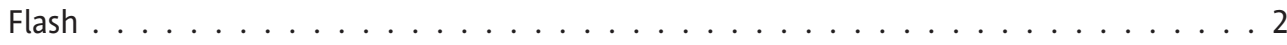

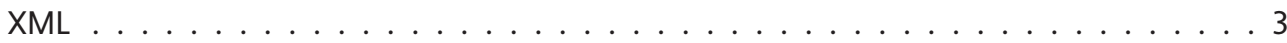

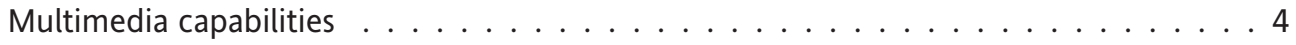

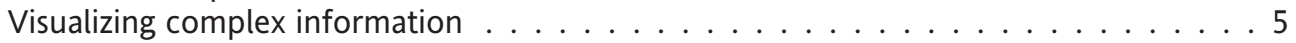

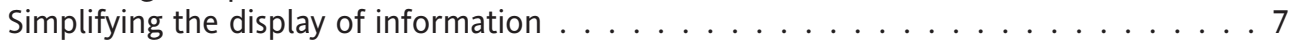

Displaying content from Office 2003 for PCs . . . . . . . . . . . . . 7

Displaying content from a web service $\ldots \ldots \ldots \ldots \ldots \ldots$

Accessing your computer . . . . . . . . . . . . . . . . 10

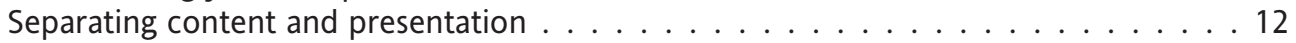

Specific applications for Flash . . . . . . . . . . . . . . . . . . . . 15

Flash as a learning tool . . . . . . . . . . . . . . . . . . . . . . 15

Creating Flash applications with Flex $\ldots \ldots \ldots \ldots \ldots \ldots \ldots$

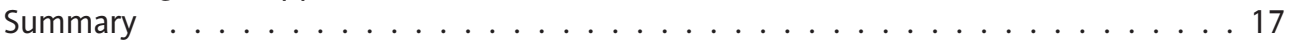


Chapter 2: Introduction to XML . . . . . . . . . . 19

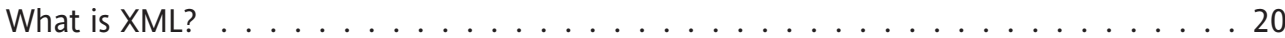

How did XML start? . . . . . . . . . . . . . . . . . . . . . 21

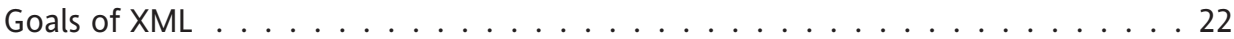

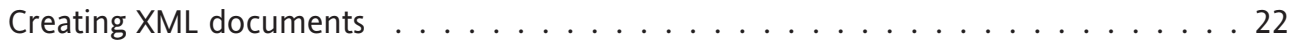

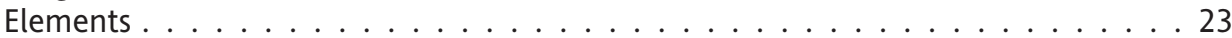

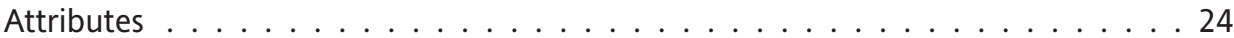

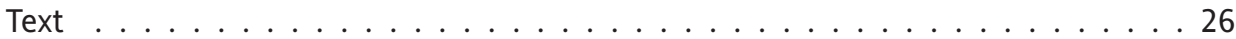

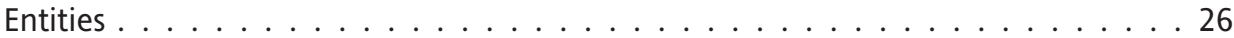

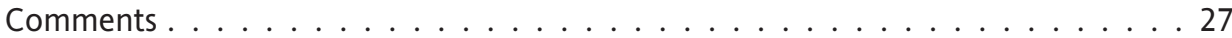

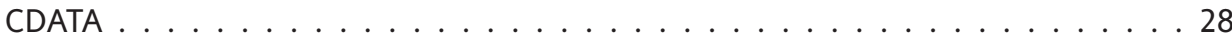

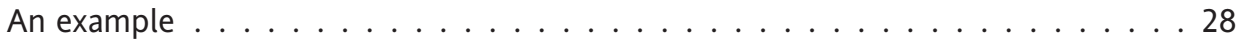

XML document parts . . . . . . . . . . . . . . . . . . . . . 28

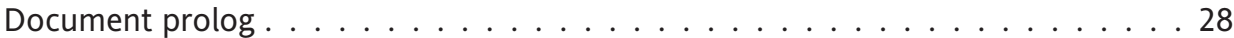

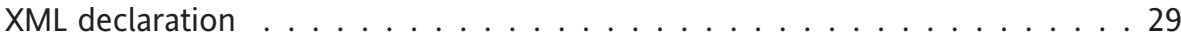

Processing instructions . . . . . . . . . . . . . . . . 29

Document Type Definitions . . . . . . . . . . . . . . . . . 30

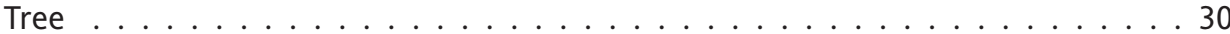

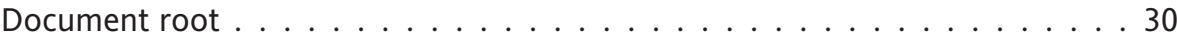

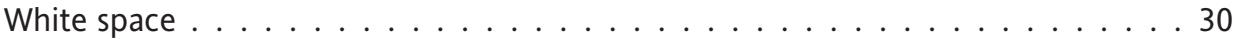

Namespaces . . . . . . . . . . . . . . . . . . . . . . . . . . 31

A simple XML document . . . . . . . . . . . . . . . . . . . . 32

Requirements for well-formed documents . . . . . . . . . . . . . . . . 34

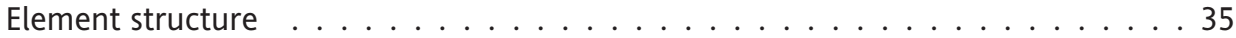

Elements must be closed . . . . . . . . . . . . . . . 36

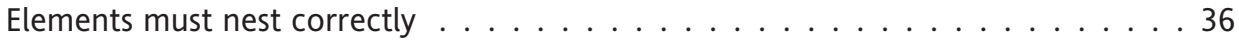

Use quotes for attributes . . . . . . . . . . . . . . . . 37

Documents that aren't well formed . . . . . . . . . . . . . . 37

Well-formed XHTML documents . . . . . . . . . . . . . . . . 38

Working with XML documents . . . . . . . . . . . . . . . 39

Generating XML content . . . . . . . . . . . . . . . . . . . . 39

Using XML information . . . . . . . . . . . . . . . . . 40

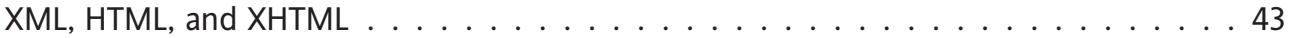

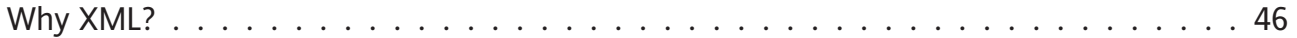

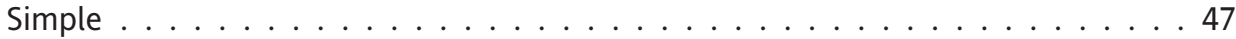

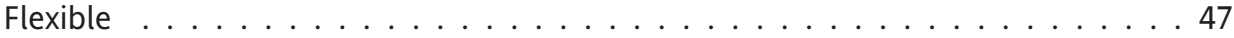

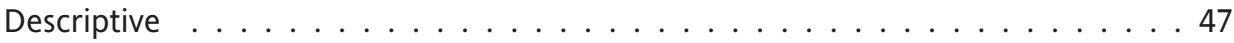

Accessible . . . . . . . . . . . . . . . . . . . . . . 48

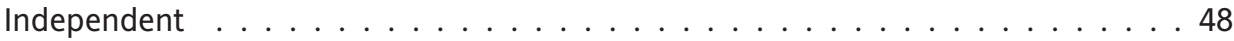

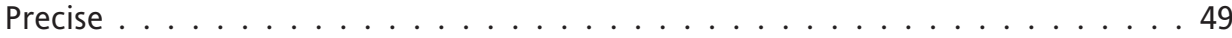

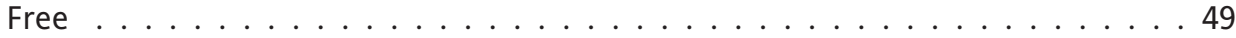

What can you do with XML? . . . . . . . . . . . . . . . . . . 50

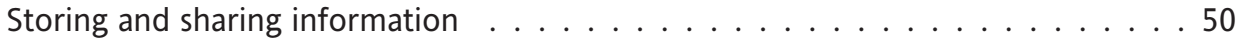

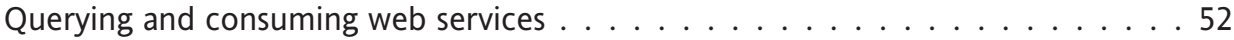

Describing configuration settings . . . . . . . . . . . . . . 52

Interacting with databases . . . . . . . . . . . . . . . 53

Interacting with Office 2003 documents . . . . . . . . . . . . . . . 53

Why is XML important to web developers? . . . . . . . . . . . . . . . . . . 54

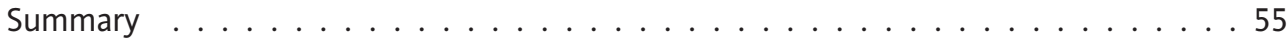


Chapter 3: XML Documents . . . . . . . . . . . 57

Creating XML content . . . . . . . . . . . . . . . . . . . . 57

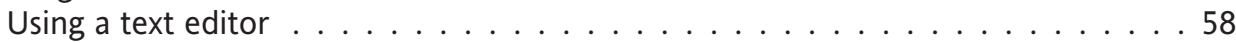

XML editors . . . . . . . . . . . . . . . . . . . . . . . . 59

Server-side files . . . . . . . . . . . . . . . . . . . . . . 64

Office $2003 / 2004 \ldots \ldots \ldots 6$

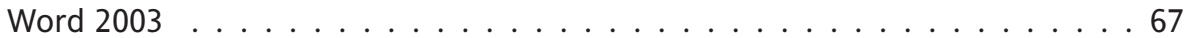

Excel . . . . . . . . . . . . . . . . . . . . . . 73

Access ............................. 80

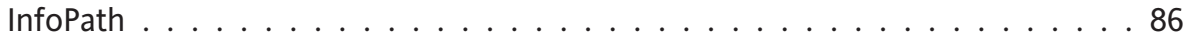

Office 2003 and data structure . . . . . . . . . . . . . . . . . 86

Consuming a web service . . . . . . . . . . . . . . . . . . 86

Using web services to interact with Amazon . . . . . . . . . . . . 87

Transforming XML content . . . . . . . . . . . . . . . . . . . 91

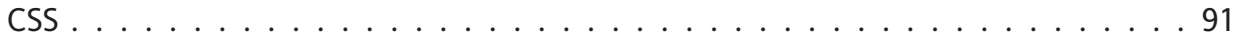

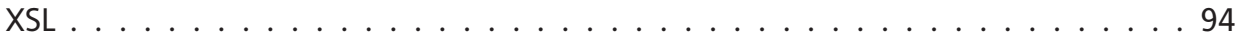

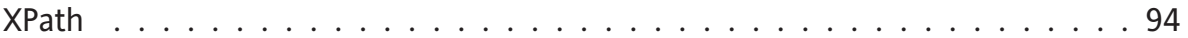

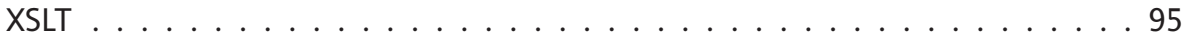

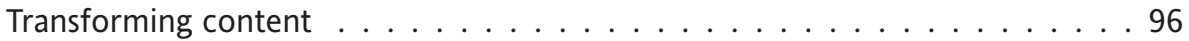

Sorting content . . . . . . . . . . . . . . . . . . 98

Filtering content . . . . . . . . . . . . . . . . . . 99

Conditional content . . . . . . . . . . . . . . . . . . 99

An example . . . . . . . . . . . . . . . . . . . . . . 999

Other methods of applying transformations . . . . . . . . . . . . . . . . 101

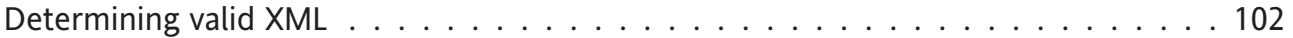

Comparing DTDs and schemas . . . . . . . . . . . . . . . . . 102

Document Type Definitions . . . . . . . . . . . . . . . . . . 103

Elements . . . . . . . . . . . . . . . . . . . . . . 104

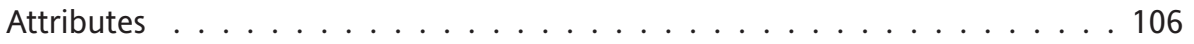

Entities . . . . . . . . . . . . . . . . . . . . . 107

A sample DTD . . . . . . . . . . . . . . . . . . . . . . . 108

XML schemas . . . . . . . . . . . . . . . . . . . . . . . . 109

Simple types . . . . . . . . . . . . . . . . . . . . . . 110

Complex types . . . . . . . . . . . . . . . . . . . . . 111

Ordering child elements . . . . . . . . . . . . . . . . . 113

Element occurrences . . . . . . . . . . . . . . . . . . . . . 113

Creating undefined content . . . . . . . . . . . . . . . . . . . 114

Annotations . . . . . . . . . . . . . . . . . . . . 114

Including a schema . . . . . . . . . . . . . . . . . . . . . 1114

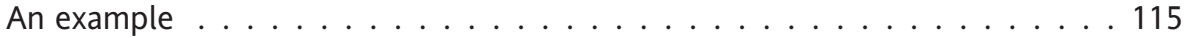

XML documents and Flash . . . . . . . . . . . . . . . . . 116

Creating an XML document . . . . . . . . . . . . . . . 116

Creating a schema . . . . . . . . . . . . . . . . . . . 120

Linking the schema with an XML document . . . . . . . . . . . . . . . . . 121

Summary . . . . . . . . . . . . . . . . . . . . . . . 122 
Chapter 4: Using the XML Class . . . . . . . . . . . . 125

Loading an XML document into Flash . . . . . . . . . . . . . . . . . . 126

Using the load method . . . . . . . . . . . . . . . . . . 126

Understanding the order of the code . . . . . . . . . . . . . . . 127

Understanding the onLoad function . . . . . . . . . . . . . . . . 128

Testing if a document has been loaded . . . . . . . . . . . . . . . . . . . . 129

Locating errors in an XML file . . . . . . . . . . . . . . . . . . . . . 130

Testing for percent loaded . . . . . . . . . . . . . . . . . . . . . . 134

Navigating an XML object . . . . . . . . . . . . . . . . . . . . . . . . 136

Mapping an XML document tree . . . . . . . . . . . . . . . 137

Understanding node types . . . . . . . . . . . . . . . . . . . . . 139

Creating node shortcuts . . . . . . . . . . . . . . . . . . . . 141

Finding the root node . . . . . . . . . . . . . . . . . . . . 141

Setting a root node variable . . . . . . . . . . . . . . . . . . . . . 142

Displaying the root node name . . . . . . . . . . . . . . . . . . . . 142

Locating child nodes . . . . . . . . . . . . . . . . . . . . . . . . 143

Working with specific child nodes . . . . . . . . . . . . . . . . . . . . 143

Working with the childNodes collection . . . . . . . . . . . . . . . . 145

Creating recursive functions . . . . . . . . . . . . . . . . . . . . . 147

Locating siblings . . . . . . . . . . . . . . . . . . . . . . . . . . 148

Locating parent nodes . . . . . . . . . . . . . . . . . . 150

Extracting information from attributes . . . . . . . . . . . . 150

Putting it all together . . . . . . . . . . . . . . . . . . . 152

Loading dynamic XML documents . . . . . . . . . . . . . . . . . . . 162

Installing IIS . . . . . . . . . . . . . . . . . . . . . 162

Creating XML content within Flash . . . . . . . . . . . . 175

Creating an XML string . . . . . . . . . . . . . . . . . 176

Creating XML using methods . . . . . . . . . . . . . . . . . . 178

Creating new elements . . . . . . . . . . . . . . . . . . 178

Creating new text nodes . . . . . . . . . . . . . . . . . . 180

Creating attributes . . . . . . . . . . . . . . . . . 182

Adding an XML declaration . . . . . . . . . . . . . . . . . . . . 182

Adding a DOCTYPE declaration . . . . . . . . . . . . . . . . . . . 183

Limits of XML methods . . . . . . . . . . . . . . . . . . . . . . . 183

Putting it all together . . . . . . . . . . . . . . . . . . . . . 184

Modifying XML content within Flash . . . . . . . . . . . . . 187

Changing existing values . . . . . . . . . . . . . . . . . . 188

Changing a text node . . . . . . . . . . . . . . . . . . 188

Changing an attribute value . . . . . . . . . . . . . . . . 189

Changing a node name . . . . . . . . . . . . . . . . . . 190

Duplicating an existing node . . . . . . . . . . . . . . . . . . . 191

Deleting existing content . . . . . . . . . . . . . . . . . . . 192

Putting it all together . . . . . . . . . . . . . . . . . . . . . . . . 194

Sending XML content from Flash . . . . . . . . . . . . . . . . . . . 197

Using the send method . . . . . . . . . . . . . . . . . . . 197

Using the sendAndLoad method . . . . . . . . . . . . . . . 200

Adding custom HTTP headers . . . . . . . . . . . . . . . . . . . 202

Putting it all together . . . . . . . . . . . . . . . . . . . . 204

Limits of the XML class in Flash . . . . . . . . . . . . . . . . 208 
No real-time interaction . . . . . . . . . . . . . . . . . 208

No validation . . . . . . . . . . . . . . . . . . . . 209

Flash cannot update external XML documents . . . . . . . . . . . . . . . . . 209

Security restrictions . . . . . . . . . . . . . . . . . . . . 209

Creating a cross-domain policy file . . . . . . . . . . . . . . . . . 209

Proxying external data . . . . . . . . . . . . . . . . . . . . 210

Tips for working with $\mathrm{XML}$ in Flash . . . . . . . . . . . . . 210

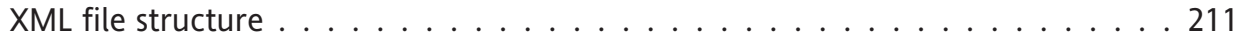

Use the XMLNode class . . . . . . . . . . . . . . . . . . . . 211

Create XML with a string . . . . . . . . . . . . . . . . . 211

Validate your XML documents . . . . . . . . . . . . . . . . . . . 211

Use the right tool for your dynamic content . . . . . . . . . . . . . . . . . 212

Summary of the properties, methods, and events of the XML class . . . . . . . . . 212

Summary . . . . . . . . . . . . . . . . . . . . . 215

Chapter 5: Working with XML in Word $2003 \ldots . . . . . .217$

Why use Microsoft Office? . . . . . . . . . . . . . . . . . . . . . . . . 218

Which Office packages can I use? . . . . . . . . . . . . . . . . . . . 219

Understanding data structures . . . . . . . . . . . . . . . . . . . . . . 219

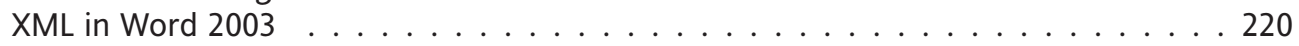

Opening an existing XML document . . . . . . . . . . . . . . 220

Transforming the document view . . . . . . . . . . . . . . . . . 221

Dealing with errors . . . . . . . . . . . . . . . . . . . . . . 224

Creating XML content with Save as . . . . . . . . . . . . . . . . . . 225

Understanding WordprocessingML . . . . . . . . . . . . . . . . . 226

Structuring content within Word . . . . . . . . . . . . . . . . . . 227

Working with the schema library . . . . . . . . . . . . . . . . . . . 228

Adding a schema to the library . . . . . . . . . . . . . . . . . . . . . . 229

Adding a transformation to a schema . . . . . . . . . . . . . . . . . 230

Creating a new Word XML document . . . . . . . . . . . . . . . . . . . 233

Attaching a schema to a Word document . . . . . . . . . . . . . . . . . 233

Adding XML tags to the document . . . . . . . . . . . . . . . . 235

Adding placeholders for empty XML tags . . . . . . . . . . . . . . . . . . 238

Adding attributes . . . . . . . . . . . . . . . . . . . . . . 241

Saving a structured XML document . . . . . . . . . . . . . . . . . . . 243

Saving transformed XML content . . . . . . . . . . . . . . . . . . . 245

Editing XML content in Word . . . . . . . . . . . . . . . . . . . . . . . . . . . . . . . . . . . . . . . . . . . . . . .

Putting it all together . . . . . . . . . . . . . . . . . . . 250

Summary . . . . . . . . . . . . . . . . . . . . 255 


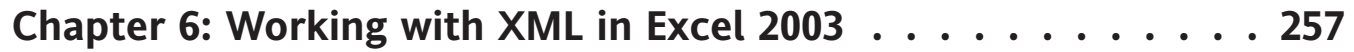

Opening an existing XML document . . . . . . . . . . . . . . . . 258

Opening as a list . . . . . . . . . . . . . . . . . . . . 258

Dealing with errors . . . . . . . . . . . . . . . . . . . 261

Opening as a read-only workbook . . . . . . . . . . . . . . . 262

Opening with the XML Source pane . . . . . . . . . . . . . . . . . 263

Opening a document with a schema . . . . . . . . . . . . . . . 265

Opening a document with an attached style sheet . . . . . . . . . . . . 265

Dealing with nonrepeating content . . . . . . . . . . . . . . 266

Dealing with mixed content . . . . . . . . . . . . . . . . . . 267

Dealing with complicated structures in a list . . . . . . . . . . . . . . . . 268

Creating XML content with Save As . . . . . . . . . . . . . . . . . . . . 272

Understanding SpreadsheetML . . . . . . . . . . . . . . . . . . . 274

Creating structured XML from an Excel document . . . . . . . . . . . . . . 275

Creating an XML map in Excel . . . . . . . . . . . . . . . . . . . 275

Adding XML elements to Excel data . . . . . . . . . . . . . . . . . . . . 277

Saving a structured XML document . . . . . . . . . . . . . . . . . . . . 279

Editing XML content in Excel . . . . . . . . . . . . . . . . . . . . . . . 284

Using the List toolbar . . . . . . . . . . . . . . . . . . . . . . . . . 284

Putting it all together . . . . . . . . . . . . . . . . . . . . 285

Summary . . . . . . . . . . . . . . . . . . . . . 289

Chapter 7: Working with XML in Access $2003 \ldots . . . . . . .291$

Exporting content as XML . . . . . . . . . . . . . . . . . . . . . . 292

Exporting a table object . . . . . . . . . . . . . . . . . . 292

Exporting a query . . . . . . . . . . . . . . . . . . . . . . . . 294

Exporting a report . . . . . . . . . . . . . . . . . . . 296

Creating a schema from Access . . . . . . . . . . . . . . . . . . 296

Creating a style sheet from Access . . . . . . . . . . . . . . . . . 298

Setting export options . . . . . . . . . . . . . . . . . . 300

Exporting linked tables . . . . . . . . . . . . . . . . . . . . 300

Applying a custom transformation . . . . . . . . . . . . . 303

Other export options . . . . . . . . . . . . . . . . . . . 306

Importing data from an external file . . . . . . . . . . . . . 308

Dealing with import errors . . . . . . . . . . . . . . . . 310

Transforming content . . . . . . . . . . . . . . . . . . . 312

Using a style sheet to import attributes . . . . . . . . . . . . . . 314

Putting it all together . . . . . . . . . . . . . . . 316

Access XML resources . . . . . . . . . . . . . . . . . . . . 322

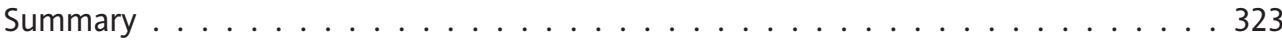


Chapter 8: Using the Data Components with XML . . . . . . 325

Understanding data components . . . . . . . . . . . . . . . . . . 325

Understanding the XMLConnector . . . . . . . . . . . . . . . . 327

Displaying read-only XML data . . . . . . . . . . . . . . . . . . . 327

Displaying updatable XML data . . . . . . . . . . . . . . . . . . . . . 328

Configuring the XMLConnector . . . . . . . . . . . . . . . . . . 328

Using the Component Inspector . . . . . . . . . . . . . . . . . . . . . . . . . 329

Creating a schema from an XML document . . . . . . . . . . . . . . 330

Creating a schema by adding fields . . . . . . . . . . . . . . . . . 331

Understanding schema settings . . . . . . . . . . . . . . . . . . . 332

Triggering the component . . . . . . . . . . . . . . . . . . . . . 334

Testing for a loaded XML document . . . . . . . . . . . . . . . . . 336

Loading an XML document into Flash . . . . . . . . . . . . . . . . . . 336

Binding XML data directly to $\mathrm{UI}$ components . . . . . . . . . . . . . 338

Adding a binding . . . . . . . . . . . . . . . . . 338

Configuring the binding . . . . . . . . . . . . . . . . 339

Using the DataSet component . . . . . . . . . . . . . . . . . . . . . 344

Binding to a Dataset component . . . . . . . . . . . . . . . . . . 345

Adding an XUpdateResolver component . . . . . . . . . . . . . . . . . . 346

Putting it all together . . . . . . . . . . . . . . . . . . . . . . . 348

The XMLConnector class . . . . . . . . . . . . . . . . . . . . . . . . . . 354

Setting the XMLConnector properties . . . . . . . . . . . . . . . . 355

Displaying the results . . . . . . . . . . . . . . . . . 355

Working with the XML class . . . . . . . . . . . . . . . . . . 356

Binding the results to components with Actionscript . . . . . . . . . . . . . 357

Including the DataBindingClasses component . . . . . . . . . . . . . . 357

Creating EndPoints . . . . . . . . . . . . . . . . . . . . . 358

Creating the binding . . . . . . . . . . . . . . . . . 358

Summary of the properties, methods, and events of the XMLConnector class . . . . 363

Summary . . . . . . . . . . . . . . . . . . . . . 365

Chapter 9: Consuming Web Services with Flash . . . . . . . 367

Consuming REST web services . . . . . . . . . . . . . . . . 369

Using REST in Flash . . . . . . . . . . . . . . . . . . . . . 370

Creating a proxy file . . . . . . . . . . . . . . . . . 370

Understanding an ASP.NET proxy file . . . . . . . . . . . . . . . . . . . 370

Consuming the XML content . . . . . . . . . . . . . . . . 371

Consuming an RSS feed . . . . . . . . . . . . . . . . . . . . . 375

Using the WebServiceConnector with SOAP web services . . . . . . . . . . . . 380

Using SOAP in Flash . . . . . . . . . . . . . . . . . . . . . . . 380

Using the WebServiceConnector . . . . . . . . . . . . . . . 380

Configuring the WebServiceConnector . . . . . . . . . . . . . . . 381

Binding the params . . . . . . . . . . . . . . . . . . . . 382

Triggering the web services call . . . . . . . . . . . . . . . . . 384

Binding the results . . . . . . . . . . . . . . . . . 386

Viewing the Web Services panel . . . . . . . . . . . . . . . . . . . . 387

Working with XML content from the WebServiceConnector . . . . . . . . . . 391 
The WebServiceConnector class . . . . . . . . . . . . . . . . . . . . . 398

Setting the WebServiceConnector properties . . . . . . . . . . . . . 398

Sending data to the web service . . . . . . . . . . . . . . . . . . . 399

Displaying the results . . . . . . . . . . . . . . . . . . . . . . 399

Summary of the properties, methods, and events of the

WebServiceConnector class . . . . . . . . . . . . . . . . . . . . . . 403

The Web Service classes . . . . . . . . . . . . . . . . . . . . . . . . . . 404

Creating a WebService object . . . . . . . . . . . . . . . . . . . . 404

Viewing the raw XML content . . . . . . . . . . . . . . . . . 405

Logging the details . . . . . . . . . . . . . . . . . . . . . 405

Summary . . . . . . . . . . . . . . . . . . . . . . 409

Chapter 10: Using the XMLSocket Class . . . . . . . . . 411

Socket server considerations . . . . . . . . . . . . . . . . . . . 412

What socket servers are available? . . . . . . . . . . . . . . . . . 412

Installing the Unity 2 socket server . . . . . . . . . . . . . . . 413

Downloading the trial version of Unity $2 \ldots \ldots \ldots . \ldots . \ldots 413$

Unpacking the unity files . . . . . . . . . . . . . . . . . . 414

Configuring the server . . . . . . . . . . . . . . . . . 414

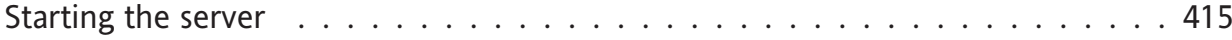

Using the XMLSocket class . . . . . . . . . . . . . . . . . . . . . 416

Creating an XMLSocket connection . . . . . . . . . . . . . 417

Sending data . . . . . . . . . . . . . . . . . . . . 419

Responding to data . . . . . . . . . . . . . . . . . . 420

Closing the connection . . . . . . . . . . . . . . . . . . . . . 420

Summary of the methods and event handlers of the XMLSocket class . . . . . . . 424

Summary . . . . . . . . . . . . . . . . . . . . . . . . 425

Chapter 11: Which XML Option Is Best for Me? . . . . . . . . . 427

Is XML the best choice? . . . . . . . . . . . . . . . . . . . . . . . . . . . . 428

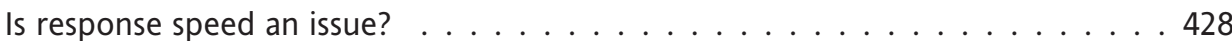

Where is the data stored? . . . . . . . . . . . . . . . . . . . . . . . 429

XML document . . . . . . . . . . . . . . . . . . . . . 429

Database . . . . . . . . . . . . . . . . . . . . . . 429

Other software package . . . . . . . . . . . . . . . . . . 440

Office 2003 document . . . . . . . . . . . . . . . . . . . . . . 430

How will the data be maintained? . . . . . . . . . . . . . . . . . . . . . . .430

Do you need server-side interaction? . . . . . . . . . . . . . . . . . . . 430

Making the decision . . . . . . . . . . . . . . . . . . . . . 431

How should you include the XML content in Flash? . . . . . . . . . . . . . . . . . 432

Using the XML class . . . . . . . . . . . . . . . . . . . . . . . . 432

Using data components . . . . . . . . . . . . . . . . . . . . . . . 432

Using the XMLConnector, WebServiceConnector, and Web Service classes . . . . . 432

Using the XMLSocket class . . . . . . . . . . . . . . . . . . . . . . . . . 433 
Making the decision . . . . . . . . . . . . . . . . . 433

Do you need real-time interaction? . . . . . . . . . . . . . . . . . 433

Is the information time sensitive? . . . . . . . . . . . . . . . . . . . . 433

Which version of Flash do you own? . . . . . . . . . . . . . . . . . 434

Which Flash players are you targeting? . . . . . . . . . . . . . . . . . . . 434

Do you prefer to work visually? . . . . . . . . . . . . . . . . . . . . . 434

A decision diagram . . . . . . . . . . . . . . . . . . . . . . . 434

Summary . . . . . . . . . . . . . . . . . . . . . . . . 436

Appendix: Useful Online Resources . . . . . . . . . . . 439

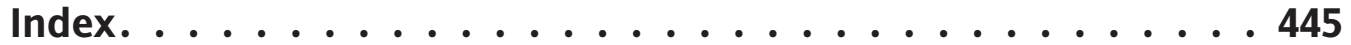




\section{FOREWORD}

If you work with Flash for anything other than creating animations, sooner or later you are going to run smack into XML. But the fact that you have this book in your hands right now means you already know this.

$\mathrm{XML}$ has been touted as the great equalizer, allowing seamless data transfer across platforms and programs. According to some, XML was going to save the world. While the world still needs some work, XML has gone a long way to improve communications between all kinds of systems.

Recognizing this some years ago, Macromedia put XML support into Flash 5, and with each version, this feature has seen remarkable improvements. If you are doing any kind of Flash application development, or even just building a dynamic website in Flash, you need to know about XML. Why? Because if you are moving data around and you don't know about XML, I can guarantee that you are wasting a lot of time and energy dealing with that data. Another reason is that, if it hasn't already happened, someday you're going to be asked to do a project that uses XML, or apply for a job where they ask you about it.

In the companies I have worked with, we always ask about a person's experience with XML and Flash. All too often the answer from beginning (and even intermediate) Flash users is something like, “XML-isn't that sort of like HTML?"

So, you need to learn XML to work with Flash. Why not just go grab any other book on XML? Well, that's what I did when I first started. They usually have a single, brush-off chapter on the makeup of XML itself, and then you start wading through DTDs, schemas, namespaces, XSLT, style sheets, and CSS, most of which doesn't have much relevance to working with XML in Flash-and most of which puts me to sleep. Moreover, the stuff you do need to know about-the Flash XML object and classes, Flash web service classes, XML sockets, and so forth-is not going to be covered by a general XML book.

However, the book you have in your hands is specifically tailored to teach you exactly what you need to know about XML for Flash applications. You are going to get a solid grounding in what XML is, why it is so cool, and how to use it in Flash. Just about every aspect of using XML as it relates to Flash is covered. Additionally, there are some very useful, real-world applications of generating XML from Microsoft Office documents. Although this may seem a bit odd at first, I can't tell you how many times l've been handed content from a client in a Word or Excel document and had to convert that to XML. (My solution has usually been to give it to an intern to convert by hand!) 
As for the author, l've known Sas for some time and can vouch for both her knowledge of the subject and for her ability to communicate and teach about it in a clear, concise, and understandable way. She has given presentations about XML at Flash conferences throughout the world, and despite the way she pronounces parse (parze) and data (dahta), she has been very well received. (Sorry, Sas, you didn't think I going to be able to resist that, did you?) In addition, she has given Flash training sessions; written tutorials, manuals, and documentation; and done technical editing for a number of books. And all that's in addition to the Flash development business she runs. In short, she knows her stuff, so listen to what she has to say!

So, fire up your computer, start Flash, get comfortable, and prepare to become a Flash XML expert!

Keith Peters

Foundation ActionScript Animation: Making Things Move!

ISBN: 1590595181

friends of ED

http://www.friendsofed.com/books/1590595181/index.html 


\section{ABOUT THE AUTHOR}

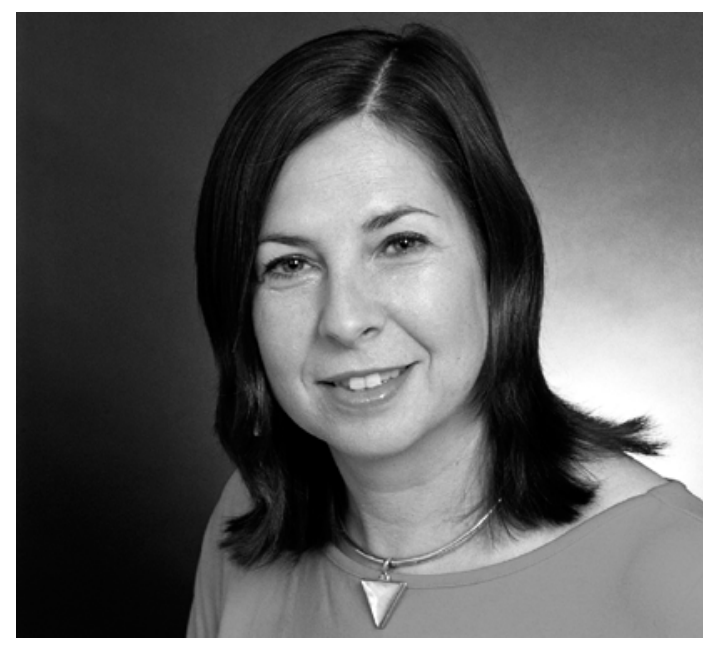

Sas Jacobs is a web developer who loves working with Flash. She set up her business, Anything Is Possible, in 1994, working in the areas of web development, IT training, and technical writing. The business works with large and small clients building web applications with ASP.NET, Flash, XML, and databases.

Sas has spoken at such conferences as Flash Forward, MXDU, and FlashKit on topics relating to XML and dynamic content in Flash.

In her spare time, Sas is passionate about traveling, photography, running, and enjoying life. One of her most fervent wishes is that Flash will take over the Web! 


\section{ABOUT THE TECHNICAL REVIEWER}

Kevin Ruse (San Jose, California) is an enthusiastic instructor who has taught at the University of California Extension Program in Cupertino, as well as DeAnza Community College. He currently provides training to Fortune 500 companies throughout the United States. He has over 20 years of experience in the graphic design and production industry, from concept to finished product. Kevin is the author of XML for Web Designers Using Macromedia Studio MX 2004 (Charles River Media, 2004) and Web Standards Design Guide (Charles River Media, 2005). 


\section{ABOUT THE COVER IMAGE}

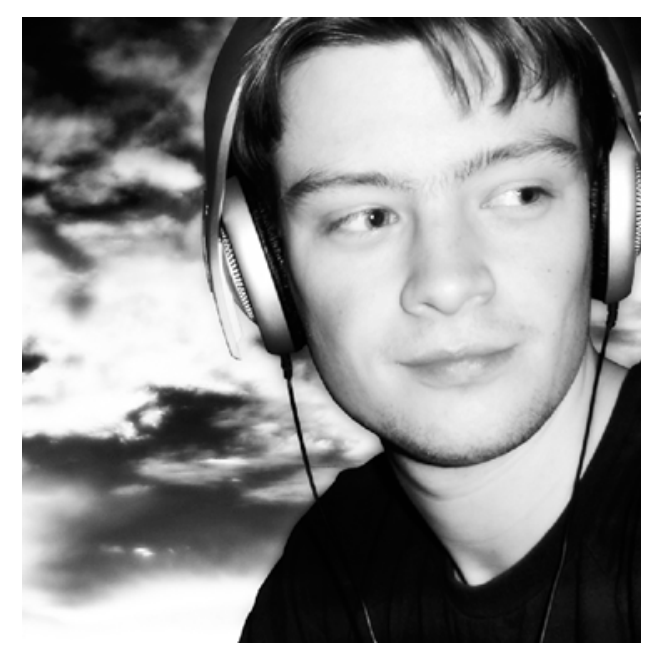

Corné van Dooren designed the front cover image for this book. Having been given a brief by friends of ED to create a new design for the Foundation series, he was inspired to create this new setup combining technology and organic forms.

With a colorful background as an avid cartoonist, Corné discovered the infinite world of multimedia at the age of 17-a journey of discovery that hasn't stopped since. His mantra has always been "The only limit to multimedia is the imagination," a mantra that is keeping him moving forward constantly.

After enjoying success after success over the past years-working for many international clients, as well as featuring in multimedia magazines, testing software, and working on many other friends of ED books-Corné decided it was time to take another step in his career by launching his own company, Project 79, in March 2005.

You can see more of Corné's work and contact him through www.cornevandooren.com or WWW. project79.com.

If you like his work, be sure to check out his chapter in New Masters of Photoshop: Volume 2, also by friends of ED (ISBN 1590593154). 


\section{ACKNOWLEDGMENTS}

I just want to say a big thank you to the people who helped in putting together this book. First, thanks to Chris Mills at friends of ED for his guidance and humor. l'd also like to thank Kevin Ruse for some great suggestions during his technical reviews. Thank you to my copy editor, Liz Welch, for her accuracy and amazing knowledge of the English language. Finally, thanks to Pat Christenson, who oversaw the whole project and made things very easy for me as a first-time author-just don't mention Chapter 4 to her! 


\section{INTRODUCTION}

This book should be subtitled "Everything you wanted to know about XML and Flash but were afraid to ask"! It introduces you to XML and shows you why, where, when, and how to use XML in Flash.

I wrote this book for both Flash designers and developers. It provides a great starting point if you haven't worked with XML before. You'll understand about the history of XML and learn about creating XML documents. I'll show you where XML fits into the web development world, and we'll look at some of the related recommendations including XPath, XML schemas, and Extensible Stylesheet Language Transformations (XSLT).

If you have Office 2003 for the PC, you'll also learn how Word, Excel, and Access 2003 can generate $\mathrm{XML}$ documents for use in Flash. Your clients can use these software packages to generate XML content for the Flash applications that you build.

The bulk of the book will work with XML in Flash. I'll show you the different ways that you can include XML content in your applications. We'll start by writing ActionScript to load and modify XML documents. Then we'll take a closer look at the data components that ship with Flash and use them with UI components like the List and DataGrid. Data components are great if you prefer to work visually. They can speed up the development process because you don't need to write any Actionscript. l'll also show you how to script these components.

The rest of the book looks at web services and XML sockets. I'll finish by showing a process that will help you to decide how to work with XML in Flash. By the end of the book, you'll have built several Flash XML applications that you'll be able to use in your work and personal projects.

The examples in the book assume a basic understanding of ActionScript, but you don't have to be an advanced coder to make them work. I've purposely kept the design of these applications very simple and used function-based code rather than ActionScript 2.0 classes. I believe this approach makes it easier for people who aren't full-time coders.

Although the book includes some server-side code examples, it doesn't aim to teach server-side coding. I'm a .NET developer so I've included VB .NET code. I've included a PHP version as well, and you can also create your own server-side files, written in other languages.

I hope you enjoy reading this book as much as I've enjoyed putting it together. During the writing process, I was continually amazed at the power of XML as a web technology. To me, the combination of Flash and XML is unbeatable, and I hope you'll discover the same thing. I see Flash as the future of the Web, and XML will help to cement that position. 


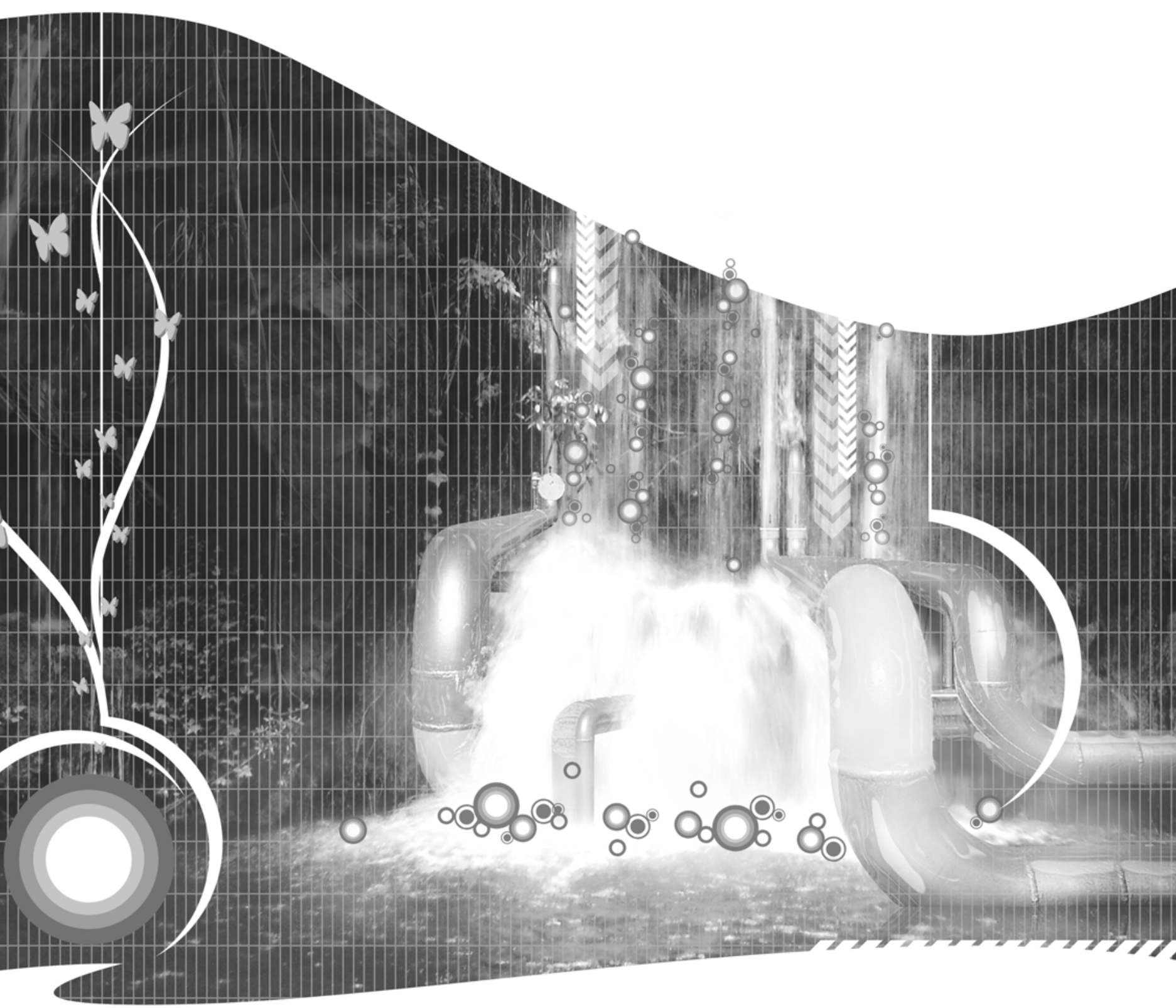

